\title{
Research on the Construction of Evaluation Index System for Chongqing International Marathon in the New Era
}

\author{
Yuqin Su $\mathbb{D},{ }^{1}$ Dalin Huang $\mathbb{D},{ }^{2}$ Zhengcheng Jin $\mathbb{D}^{1},{ }^{1}$ Shifan Chen $\mathbb{D},{ }^{3}$ and Ao Chen $\mathbb{D}^{4}$ \\ ${ }^{1}$ College of Physical Education, Chongqing University of Posts and Telecommunications, Chong'qing 400065, China \\ ${ }^{2}$ College of Physical Education, Chongqing Mechanical and Electrical Vocational Technical University, Chong'qing 400065, China \\ ${ }^{3}$ Electrical Engineering and Automation Department, \\ College of Automation, Chongqing University of Posts and Telecommunications, Chong'qing 400065, China \\ ${ }^{4}$ Internet of Things Engineering Department, College of Automation, Chongqing University of Posts and Telecommunications, \\ Chong'qing 400065, China
}

Correspondence should be addressed to Yuqin Su; 2018213105@stu.cqupt.edu.cn

Received 11 October 2021; Revised 18 November 2021; Accepted 29 November 2021; Published 16 December 2021

Academic Editor: Sang-Bing Tsai

Copyright (c) 2021 Yuqin Su et al. This is an open access article distributed under the Creative Commons Attribution License, which permits unrestricted use, distribution, and reproduction in any medium, provided the original work is properly cited.

Chongqing International Marathon event is one of the top marathon events in China. Under the background of the new era, constructing a scientific and reasonable marathon evaluation system plays an important role in the development of this event. In this study, literature method, field investigation method, Delphi method, interview method, analytic hierarchy process, and other methods are used. And it includes 5 primary indicators, 13 secondary indicators, and 58 tertiary indicators. What is more, AHP is used to determine the weight of indicators at each level. This evaluation system can help the race-organizing committee more scientifically understand this event and lay a solid foundation for finally building an excellent brand event.

\section{Introduction}

Statistics from the German Athletics Association show that there are 180 professional marathons and 3,700 mass marathons in Germany every year. Every week, there are thousands of free marathons in Germany. Currently, more than 100 cities in Germany hold New Year's Eve Marathon or New Year's Marathon. The events of the competition range from 800 meters to 20 kilometers. Contestants range from kindergarten children to grayhaired elderly people. After 11 years of development and precipitation, Chongqing International Marathon [1] (P.115), as one of the top marathon events in Chongqing and even in China, has attracted extensive attention from the whole society.

With the development of the economy and society, people pay more and more attention to health, and more people participate in sports. Running is the most and convenient choice. Chongqing International Marathon is one of the more famous marathon events in China and even in the world. The projects are diverse and distinctive, and the number of participants has doubled year by year. According to statistics, the 2019 Chongqing International Marathon has four events: full course, half course, minimarathon, and parent-child marathon, with a total enrollment of more than 100,000. Runners from more than 50 countries and regions and more than 500 cities all over the world participated in the forecast, with more than 30,000 participants and 220 foreign athletes. However, due to the need for epidemic prevention and control, the scale of the Chongqing International Marathon in 2020 will be adjusted from 30,000 to less than 4,900, including 4,000 full marathons and 900 minimarathons, and the parent-child project will be canceled. It can be seen that the Chongqing International Marathon is deeply loved by people.

The core of the Chongqing International Marathon is a sports competition. Because the event is held in Chongqing, 
it has always been a characteristic event of Chongqing mountain city and river since its establishment. Therefore, building such a marathon brand event in line with Chongqing will have a positive impact on the regional politics, economy, culture, society, and environment and will also become a landmark social activity in Chongqing.

As Chongqing has gradually become one of the most popular tourist cities in China, more and more tourists begin to choose sports elements in tourism. "Sports + tourism" is becoming a popular new leisure lifestyle, which also makes the sports development of Chongqing to enter the fast lane. The holding of the Chongqing International Marathon is of positive significance to Chongqing's economic development, cultural development, and sports development. Therefore, more and more tourists also come to Chongqing.

Both the quality of the event and the number of participants showed a geometric growth [2]. Although China's marathon events have rapidly developed in recent years, many problems still occur from time to time, such as the overall growth stage, the imperfect event mechanism, the relatively insufficient operation and management experience of event personnel, and the weak awareness of the organizing committee to scientifically run events. This is because it has not formed a relatively complete event index evaluation system [3] (P.67-76) as a reference standard for the comprehensive evaluation of the event-organizing committee to help the better development of the event. The event index evaluation system can be used as a reference standard to evaluate whether the overall operation mechanism of an event is excellent or not, and it is also a basic evaluation standard for whether an event can become a brand event [4]. At present, there is an extreme lack of relevant research on the evaluation index system of marathon in China. A total of 1,870 articles were retrieved by inputting the keyword "marathon" in CNKI literature retrieval. In the search keyword "sports event evaluation index system," there are only 46 results, but there are only two articles in the further search keyword "marathon event evaluation system." Although the Chongqing International Marathon has achieved certain success after year-on-year development, it still lacks an effective event evaluation index system to guide its future development direction and help the Chongqing International Marathon make experience summary, and the establishment of an event evaluation index can well fill this gap. Based on this, it is urgent to establish a scientific and effective evaluation index system [5] of the Chongqing International Marathon.

In this study, we have built a complete evaluation index system for the Chongqing International Marathon brand event. As an international marathon gold medal event, the event has a very good reputation at home and abroad. As one of China's four municipalities directly under the central government, Chongqing has an important economic and social status, and the development of large-scale mass sports events is also a research hotspot in recent years. On the one hand, the construction of the evaluation index system can comprehensively evaluate the effectiveness of the event and provide important theoretical support for the sustainable development of the event. On the other hand, it also has universality. On this basis, it can provide reference and reference for other marathon events at home and abroad. The members of the research group personally participated in the preparation and holding of the event in the early stage and successfully applied for the scientific research project of Chongqing Sports Bureau.

Most of the existing marathon competition evaluation systems only use SWOT analysis and literature methods for research. We extend and add the method to design a more detailed evaluation system.

At the same time, the survey objects selected by the Delphi method are also experts and scholars with important influence in Chinese sports academic circles. Their support for the subject makes the results of the whole study more scientific and rigorous.

\section{Research Methods}

2.1. Documentation Method. According to keywords and research directions, we consulted many books on sports events, sports social humanities, sports management, sports economics, sports event evaluation, and read relevant studies such as sports event evaluation, event evaluation system, event evaluation indicators, event evaluation standards, and event evaluation construction through CNKI. Using academic search engines such as Scirus, Issuu, CNKI, and Google, keywords such as "marathon event evaluation," "marathon event organization and management," "marathon brand event evaluation," and "evaluation index system" are input. On this basis, the primary, secondary, and tertiary evaluation index factors of recelebrating the international marathon brand event are preliminarily screened.

\subsection{Investigation Method}

2.2.1. Interview Method. By visiting experts and scholars in the fields of domestic sports event organization and management, event evaluation standards, sports event evaluation system research, marathon event operation and management, and visiting relevant leaders and staff of Chongqing Chongma Sports Development Co., Ltd., we learned about the opinions of relevant personnel on the evaluation index system of the Chongqing International Marathon brand events and further improved the scientificity and preciseness of the evaluation index system of the Chongqing International Marathon brand events.

2.2.2. Delphi. We designed the expert interview outline of the Chongqing International Marathon brand event evaluation system, the screening questionnaire for the importance of indicators at all levels and the Chongqing International Marathon brand event evaluation questionnaire (the questionnaire is tested by experts for reliability and validity and meets the statistical requirements, and the experts modify the evaluation indicators and assign the index weight). The overall survey is distributed, recovered, and distributed in two rounds according to the procedure 
after sorting out, and the expert opinions basically tend to be consistent.

Following the principles of anonymity, feedback, statistics, and research needs of the Delphi method [6], 15 experts and scholars were selected to conduct the second round of questionnaire survey, screening, and determine the index system, including 4 sports experts; 3 sports management experts; 4 sports sociology experts; 2 government staff; and 2 persons from Chongqing Chongma Sports Development Co., Ltd. (see Table 1 for details).

2.3. Hierarchical Analysis. Analytic hierarchy process [7] (P.148-153) (AHP) decomposes the problem into different constituent factors according to the nature of the problem and the overall goal to be achieved and aggregates and combines the factors according to different levels according to the correlation, influence, and subordinate relationship between the factors to form a multilevel analysis structure model. Thus, the problem is finally attributed to the determination of the relatively important weight of the lowest level (schemes and measures for decision-making) relative to the highest level (overall goal) or the arrangement of the relative advantages and disadvantages. This study establishes a multilevel rating index model of sports events by using the AHP method, then gives a quantitative description of the relative importance of each level of evaluation index according to expert advice, finally, determines the value of the relative importance order of all indicators at each level, and conducts consistency test. If the consistency conditions are not met, the judgment matrix is modified until they are met.

2.4. Logical Analysis Method. On the basis of combining a large number of theoretical data and mathematical data, through logic demonstration and summary, the evaluation index system of the Chongqing International Marathon brand events has been initially established.

2.5. Mathematical Statistics Method. This study obtained the analysis of the expert questionnaire, the factor analysis, and the quantitative calculation. It lays a foundation for the construction of Chongqing International Marathon evaluation index system.

\section{Establishment Process of the Evaluation Index System of the Chongqing International Marathon Brand Events}

The establishment of the Chongqing International Marathon brand event index system is a complex and detailed process. We need to first establish a basic understanding of the research object, then conduct an overall feature analysis of the research object, then model the research object and conduct in-depth research, then gradually improve the existing research, and finally form a systematic research result [8] (P.9-13). Therefore, the research process of this study is roughly divided into four steps: theoretical preparation, preliminary selection of index system, test of index factors and determination of weight, and establishment of evaluation system of the Chongqing International Marathon brand event (see Figure 1 for details). In the research method, the analytic hierarchy process is mainly used, because other analysis methods such as principal component analysis are not applicable to this study. Taking principal component analysis as an example, it needs to ensure that the cumulative contribution rate of the first few principal components extracted reaches one, and the high-level and extracted principal components must be able to give explanations that conform to the actual background and meaning.

\section{Construction of the Evaluation Index System of the Chongqing International Marathon Brand Events}

4.1. Preliminary Construction Process of the Construction of the Index System. Through the field investigation on the overall history, organization and holding, politics, economy, society, humanities, environment, and other factors of the Chongqing International Marathon, and by consulting the studies of China Journal Network on events, marathon, evaluation index system, and so on, and by studying the laws and regulations on sports events such as the 14th five-year plan for sports development [9] of government departments, it can be found that the "system" theory [10] (P.70-78) is a theoretical method suitable for constructing the evaluation index system of the Chongqing International Marathon. The word "system" originated from ancient Greek, meaning that parts constitute a whole. It was not until L. von Bertalanffy founded the "general system theory" [11] (P.5-8) in 1937. L. von. Bertalanffy believes that it is inappropriate to limit the general system theory to technology as a mathematical theory, because there are many systemic problems that cannot be expressed by modern mathematical concepts. The term general system theory has a broader content, including a very wide range of research fields and three main aspects: first, the science of system, also known as mathematical system theory. This is to use accurate mathematical language to describe the system and study the fundamental theory applicable to all systems. Second is system technology, also known as system engineering. This is to study complex systems such as engineering system, life system, economic system, and social system with system thought and system method. Third is system philosophy: it studies the nature of the scientific methodology of general system theory and raises it to the status of philosophical methodology. L. Von. Bertalanffy tried to extend the general system theory to the category of system science, including almost all three levels of system science. However, the main research contents of modern general system theory are still limited to system thought, system isomorphism, open system, and system philosophy.

The subsystems involved in large-scale sports events such as the Chongqing International Marathon include politics, economy, culture, society, and environment. These 
TABLE 1: List of experts $(n=15)$.

\begin{tabular}{lcc}
\hline Expert category & Professional title (number) & Degree (number) \\
\hline Sports experts & Professor(1); associate professor (3) & Doctor(3); master(1) \\
Sociology of sports humanities & Professor(1); associate professor (3) & Doctor(3); master(1) \\
Sports event management experts & Professor(1); associate professor (3) & Doctor(3); master(1) \\
Staff of relevant government departments & Deputy director general (1); section chief(1) & Master(1); bachelor (1) \\
Chongqing Chongma Sports Development Co., & Department general manager (1); clerk general & Bachelor (2) \\
Ltd. & $(1)$ & Doctor (9); master (3); bachelor (3) \\
Total number & 15 &
\end{tabular}

systems are closely interrelated. For the Chongqing International Marathon, it itself is a huge system engineering . The index evaluation of system engineering needs to be considered from the perspective of system, its operation and development must follow the viewpoint of system theory, and its internal subsystems and internal factors independently interact and develop. Therefore, it is necessary to analyze the political, economic, cultural, social, and environmental systems involved in the event and then systematically analyze each subsystem on the basis of the above analysis to obtain the index factors of each subsystem.

Finally, considering the time and economic cost of the survey, and the factors of reasonable selection of indicators, high evaluability, and high testability, the evaluation index system [12] (Table 2) of the Chongqing International Marathon brand event is preliminarily determined, which is composed of five first-class indicators [13], 13 second-class indicators, and 58 third-class indicators, including politics, economy, culture, society, and environment.

\subsection{Selection Process of the Index System}

4.2.1. First Round of Expert Consultation. First, this study carried out expert thematic discussion and open-ended questionnaire survey on the rationality of the primary indicators. The result is that the concentration of reservations of the five primary indicators is relatively high, so the primary indicators do not need to be modified. Then, according to the preliminary index system, the questionnaire is made into two sections: secondary index and tertiary index I. The scoring mechanism of the questionnaire adopts the basic idea of the Likert 5 scale method [14] (P.18-23,28), that is, the expert score is graded according to important-unimportant 5. However, considering the traditional the Likert 5 scale is rough for complex systems such as medium and large marathon systems, it is improved to a continuous graduation scale. SPSS 26.0 was used to analyze the questionnaire I. We use Cronbach's $\alpha$ to test the reliability of the scale. If the $\alpha$ coefficient does not exceed 0.6 , it is generally considered that the internal consensus reliability is insufficient; when it reaches $0.7-0.8$, it means that the scale has considerable reliability, and when it reaches $0.8-0.9$, it means that the scale's reliability is very good. The result is that Cronbach's $\alpha$ is 0.81 , which indicates that the reliability of the scale is good. Through letters, questionnaires, and face-to-face interviews, questionnaires were collected on the spot, and 15 valid questionnaires were obtained. SPSS 26.0 was used for analysis to determine whether the index was retained or not according to the importance mean, standard deviation, and coefficient of variation of each index score. Among them, the mean value of importance reflects the unified result of expert opinions. The greater the value, the more the index should be retained. The standard deviation reflects the divergence degree of expert opinions. The smaller the value, the smaller the divergence caused by the index, and the more the index should be retained. The smaller the coefficient of variation of each index score, the higher the coordination of expert opinions, and the more the index should be retained. We eliminated the six tertiary indicators with the smallest mean value, the largest standard deviation, and the largest coefficient of variation and retained 13 secondary indicators and 52 tertiary indicators (see Tables 3 and 4 for the scores and screening results of secondary and tertiary indicators). In Table 3, secondary indicators correspond to each support item under primary indicators in Table 2. The results are discussed with experts after the following revisions: (1) merge C49 into C21; (2) simplify C54 to environmental protection facilities.

4.2.2. Second Round of Expert Consultation. According to the results of the first round of expert consultation, a questionnaire II containing secondary indicators and tertiary indicators is formulated. The scoring mechanism of the questionnaire still adopts the improved Likert 5 scale method II, the Krambaha reliability coefficient is 0.85 , indicating that the scale has good reliability. Questionnaire II is issued to experts, and the questionnaire was analyzed according to the same method as in round 1 of II and followed the opinions of experts and imposed more strict restrictions on the mean value of importance. When the mean value of importance is less than $75 \%$ (i.e., 3.75) of the full score, it is considered that the index cannot meet the standard. In the second round, one secondary indicator and 14 tertiary indicators were successively deleted, and finally an indicator system with 12 secondary indicators and 37 tertiary indicators was obtained (see Table 5). The expert opinions of the indicator system were highly unified. The complete index construction process is shown in Figure 2.

4.3. Weight Determination of the Index System. Whether the index weight [15] (P.47-55) is reasonable greatly affects the scientificity and correctness of the evaluation. In this study, AHP and probability distribution function are preselected to calculate the weight of each index. The two methods will be discussed with experts. Considering the AHP method, 


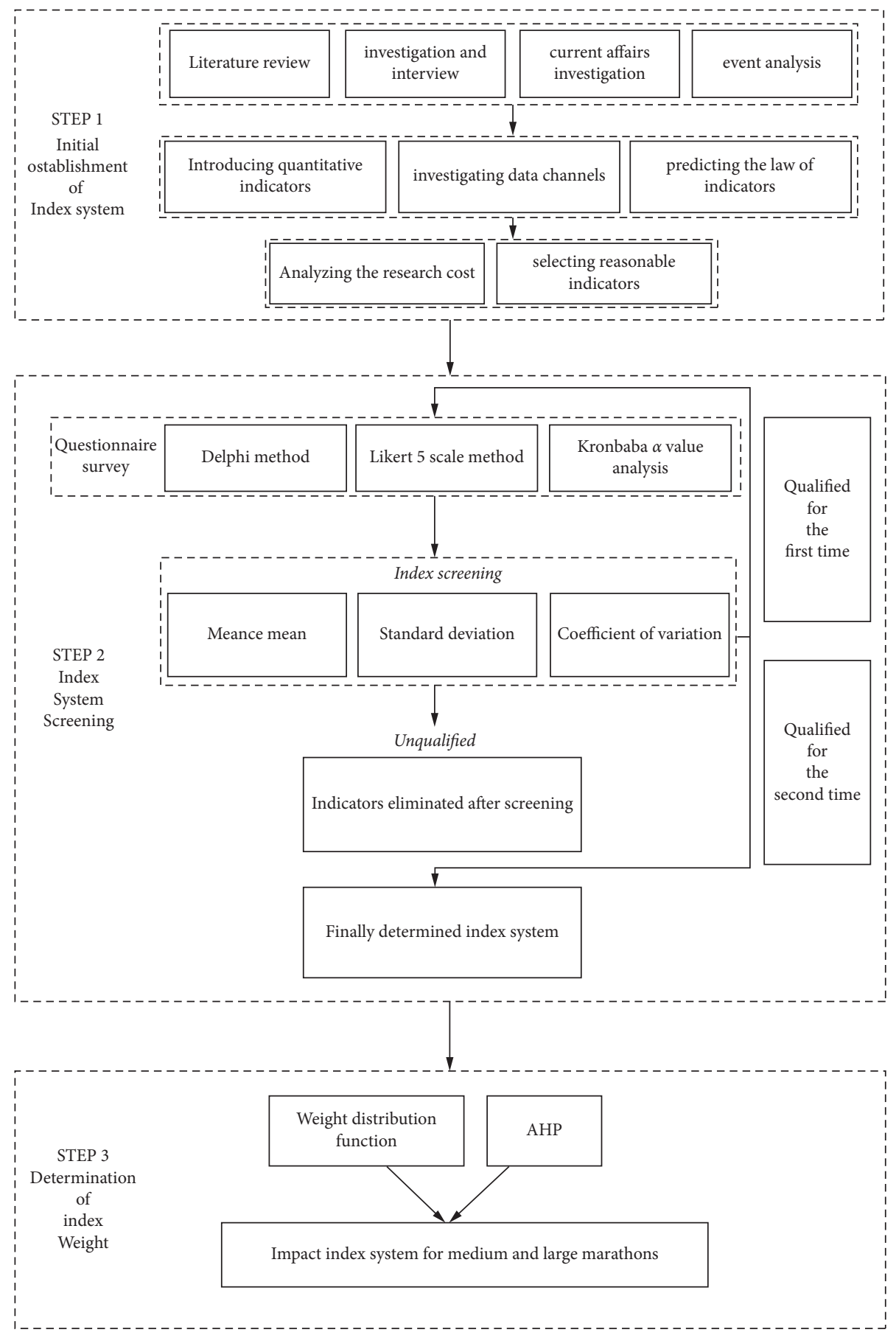

Figure 1: The establishment process of the evaluation index system of the Chongqing International Marathon brand competition.

experts need to be reinvited to compare and fill in the form for up to 2,275 times among 5 primary indicators, $12 \mathrm{sec}$ ondary indicators, and 37 tertiary indicators, which will waste experts' energy cost and research time cost. The improved Likert 5 scale method has good perception and accuracy for quantitative scoring. Therefore, the latter probability distribution function is used to calculate the weight of each index. Among them, the weight of each threelevel indicator is the average value of its importance in the second round of expert consultation. The weight $X$ of level I and level II indicators obeys uniform distribution $X \sim U(a, b)$, and its calculation formula is 
Table 2: Preliminary index system.

\begin{tabular}{ll}
\hline $\begin{array}{l}\text { Primary } \\
\text { indicators }\end{array}$ & Secondary indicators \\
\hline Polity A1 & Policy implementation B1
\end{tabular}

Regional exchanges B2

Positive economic impact B3

Economy A2

Negative economic impact B4

\author{
Tertiary indicators
}

The practice degree of the new pattern of the competition $\mathrm{C} 2$

The intensity of sports event construction in a strong sports city $\mathrm{C} 3$

The construction intensity of strengthening the city with sports talents C4

To welcome the centenary anniversary of the founding of the communist party of China C5

Construction along the "one belt, one road" line C6 Regional event exchange C7

Tourism gain C8

Regional per capita sports GDP C9

Local event organizers $\mathrm{C} 10$

Economic benefits of regional characteristic brand C11

Positive impact on the talent market $\mathrm{C} 12$

Positive impact on tourism $\mathrm{C} 13$

The GDP value C14

The event department a loss C15

Foreign goods encroach on the market C16

Negative impact on the talent market C17

Event loss C18

Destruction of commodity market value system C19

Negative impact on the operation of the scenic spots along the way C20
Cultural improvement B5

Culture A3

Carry forward the

characteristics B6
Carry forward the Chinese spirit of sports C21
Building the socialist core value system C22

Training of local sports stars C23

The development of sports spirit and culture C24

Event-related cultural events C25

Publicity of the featured events C26

Design of characteristic medals C27

Design of characteristic competition route C28

Setting of characteristic events C29

Display of regional culture C30
Regional prestige B7

Event visibility B8

Society A4

Regional sports popularization

B9

Resident life B10

Educational diffusion effect

B11

The degree of influence on stimulating the students' will and quality and establishing the lifelong exercise goal C49
Number of heavy horse research topics C48

Media publicity $\mathrm{C} 32$

Athletes' satisfaction C33

Sponsor SatisfactionC34

Number of contestants level C35

Level of the contestant C36

Media quantity and level C37

Regional marathon participation C38

Regional sports event participation C39

Regional citizens pay attention to the ways $\mathrm{C} 40$

Regional citizens' sports literacy training C41

Level of urban sports public service C42

Safety misadventure C43

Traffic interference C44

Life satisfaction of the residents C45

Integration of university marathon courses C46

Research on university marathon topics C47
Positive environmental impact

Environment A5

Implementation of precompetition environmental publicity C50

Environmental remediation caused by the event C51
The implementation of the event in environmental demonstration C52

Promotion of the event to local residents' awareness of environmental protection C53

Promotion degree of greening design and construction C54

Degree of water damage in the competition C55

Negative environmental impact B13

Degree of damage of the surrounding living environment C56

Waste cleaning efforts C57

Energy consumption degree of the event C58 
TABLE 3: Mean and coefficient of variation of the secondary indicators.

\begin{tabular}{|c|c|c|c|c|}
\hline Secondary indicators & Mean of importance & Standard deviation & Coefficient of variation & Result \\
\hline Policy implementation B1 & 4.25 & 0.42 & 0.10 & Retaining \\
\hline Regional exchanges B2 & 3.58 & 1.43 & 0.40 & Retaining \\
\hline Positive economic impact B3 & 4.17 & 0.75 & 0.18 & Retaining \\
\hline Negative economic impact B4 & 3.83 & 0.98 & 0.26 & Retaining \\
\hline Cultural improvement B5 & 3.83 & 0.98 & 0.26 & Retaining \\
\hline Carry forward the characteristics B6 & 4.17 & 0.75 & 0.18 & Retaining \\
\hline Regional prestige $\mathrm{B} 7$ & 4.08 & 0.92 & 0.22 & Retaining \\
\hline Event visibility $\mathrm{B} 8$ & 4.75 & 0.42 & 0.09 & Retaining \\
\hline Regional sports popularization B9 & 4.00 & 0.63 & 0.16 & Retaining \\
\hline Resident life B10 & 4.00 & 0.89 & 0.22 & Retaining \\
\hline Educational diffusion effect $\mathrm{B} 11$ & 4.67 & 0.52 & 0.11 & Retaining \\
\hline Positive environmental impact B12 & 3.50 & 1.22 & 0.35 & Retaining \\
\hline Negative environmental impact B13 & 3.83 & 1.17 & 0.30 & Retaining \\
\hline
\end{tabular}

TABLE 4: Mean and coefficient of variation of the tertiary indicators.

\begin{tabular}{|c|c|c|c|c|}
\hline Tertiary indicators & $\begin{array}{l}\text { Meance } \\
\text { mean }\end{array}$ & $\begin{array}{l}\text { Standard } \\
\text { deviation }\end{array}$ & $\begin{array}{l}\text { Coefficient of } \\
\text { variation }\end{array}$ & Result \\
\hline Implementation degree of national fitness $\mathrm{C} 1$ & 4.14 & 0.84 & 0.20 & Retaining \\
\hline The practice degree of the new pattern of the competition $\mathrm{C} 2$ & 3.96 & 0.63 & 0.16 & Retaining \\
\hline The intensity of sports event construction in a strong sports city C3 & 3.93 & 0.83 & 0.21 & Retaining \\
\hline The construction intensity of strengthening the city with sports talents $\mathrm{C} 4$ & 3.13 & 0.83 & 0.27 & Retaining \\
\hline $\begin{array}{l}\text { To welcome the centenary anniversary of the founding of the communist } \\
\text { party of China C5 }\end{array}$ & 3.18 & 1.07 & 0.34 & Retaining \\
\hline Construction along the "one belt, one road" line C6 & 3.89 & 0.96 & 0.25 & Retaining \\
\hline Regional event exchange C7 & 4.46 & 0.50 & 0.11 & Retaining \\
\hline Tourism gain $\mathrm{C} 8$ & 3.32 & 0.77 & 0.23 & Retaining \\
\hline Regional per capita sports GDP C9 & 4.21 & 0.67 & 0.16 & Retaining \\
\hline Local event organizers $\mathrm{C} 10$ & 4.57 & 0.51 & 0.11 & Retaining \\
\hline Economic benefits of regional characteristic brand $\mathrm{C} 11$ & 3.25 & 0.75 & 0.23 & Retaining \\
\hline Positive impact on the talent market $\mathrm{C} 12$ & 2.39 & 1.15 & 0.48 & Retaining \\
\hline Positive impact on tourism $\mathrm{C} 13$ & 2.43 & 1.02 & 0.42 & Delete \\
\hline The GDP value $\mathrm{C} 14$ & 2.29 & 1.20 & 0.53 & Delete \\
\hline The event department a loss C15 & 3.25 & 0.75 & 0.23 & Retaining \\
\hline Foreign goods encroach on the market $\mathrm{C} 16$ & 3.29 & 0.80 & 0.24 & Delete \\
\hline Negative impact on the talent market C17 & 3.43 & 1.07 & 0.31 & Retaining \\
\hline Event loss C18 & 3.93 & 0.83 & 0.21 & Delete \\
\hline Destruction of commodity market value system C19 & 3.39 & 0.88 & 0.26 & Retaining \\
\hline Negative impact on the operation of the scenic spots along the way C20 & 4.20 & 0.68 & 0.16 & Retaining \\
\hline Carry forward the Chinese spirit of sports C21 & 3.82 & 1.14 & 0.30 & Retaining \\
\hline Building the socialist core value system C22 & 4.21 & 0.70 & 0.17 & Retaining \\
\hline Training of local sports stars C23 & 3.64 & 1.15 & 0.32 & Retaining \\
\hline The development of sports spirit and culture C24 & 3.68 & 1.10 & 0.30 & Retaining \\
\hline Event-related cultural events C25 & 4.07 & 1.00 & 0.24 & Retaining \\
\hline Publicity of the featured events C26 & 4.00 & 0.76 & 0.19 & Retaining \\
\hline Design of characteristic medals C27 & 4.14 & 0.74 & 0.18 & Retaining \\
\hline Design of characteristic competition route $\mathrm{C} 28$ & 3.82 & 1.07 & 0.28 & Retaining \\
\hline Setting of characteristic events C29 & 4.14 & 0.53 & 0.13 & Retaining \\
\hline Display of regional culture C30 & 4.32 & 0.72 & 0.17 & Retaining \\
\hline Event level C31 & 4.29 & 0.73 & 0.17 & Retaining \\
\hline Media publicity C32 & 4.27 & 0.71 & 0.17 & Retaining \\
\hline Athletes' satisfaction C33 & 3.99 & 0.66 & 0.17 & Retaining \\
\hline Sponsor satisfaction C34 & 3.14 & 0.84 & 0.27 & Retaining \\
\hline Number of contestants level C35 & 3.54 & 0.80 & 0.23 & Retaining \\
\hline Level of the contestant C36 & 3.70 & 0.58 & 0.16 & Retaining \\
\hline Media quantity and level C37 & 2.21 & 1.12 & 0.51 & Retaining \\
\hline Regional marathon participation C38 & 2.71 & 1.33 & 0.49 & Retaining \\
\hline Regional sports event participation C39 & 2.86 & 0.95 & 0.33 & Retaining \\
\hline
\end{tabular}


TABle 4: Continued.

\begin{tabular}{|c|c|c|c|c|}
\hline Tertiary indicators & $\begin{array}{l}\text { Meance } \\
\text { mean }\end{array}$ & $\begin{array}{l}\text { Standard } \\
\text { deviation }\end{array}$ & $\begin{array}{l}\text { Coefficient of } \\
\text { variation }\end{array}$ & Result \\
\hline Regional citizens pay attention to the ways $\mathrm{C} 40$ & 3.41 & 1.07 & 0.31 & Retaining \\
\hline Regional citizens's sports literacy training C41 & 3.71 & 0.91 & 0.25 & Retaining \\
\hline Level of urban sports public service C42 & 3.26 & 0.86 & 0.26 & Retaining \\
\hline Safety misadventure C43 & 3.21 & 1.12 & 0.35 & Retaining \\
\hline Traffic interference C44 & 3.47 & 0.90 & 0.26 & Retaining \\
\hline Life satisfaction of the residents $\mathrm{C} 45$ & 3.39 & 0.88 & 0.26 & Retaining \\
\hline Integration of university marathon courses C46 & 3.27 & 1.31 & 0.40 & Retaining \\
\hline Research on university marathon topics C47 & 2.99 & 1.01 & 0.34 & Retaining \\
\hline Number of heavy horse research topics C48 & 3.36 & 1.15 & 0.34 & Retaining \\
\hline $\begin{array}{l}\text { The degree of influence on stimulating the students' will and quality and } \\
\text { establishing the lifelong exercise goal C } 49\end{array}$ & 1.89 & 0.84 & 0.44 & Retaining \\
\hline Implementation of precompetition environmental publicity C50 & 1.96 & 0.80 & 0.41 & Retaining \\
\hline Environmental remediation caused by the event C51 & 2.36 & 0.74 & 0.32 & Retaining \\
\hline The implementation of the event in environmental demonstration C52 & 2.04 & 1.25 & 0.61 & Retaining \\
\hline $\begin{array}{l}\text { Promotion of the event to local residents' awareness of environmental } \\
\text { protection C53 }\end{array}$ & 4.14 & 0.84 & 0.20 & Retaining \\
\hline Promotion degree of greening design and construction C54 & 3.96 & 0.63 & 0.16 & Retaining \\
\hline Degree of water damage in the competition C55 & 3.93 & 0.83 & 0.21 & Delete \\
\hline Degree of damage of the surrounding living environment C56 & 2.96 & 0.77 & 0.26 & Retaining \\
\hline Waste cleaning efforts C57 & 2.79 & 0.70 & 0.25 & Retaining \\
\hline Energy consumption degree of the event C58 & 3.89 & 0.96 & 0.25 & Delete \\
\hline
\end{tabular}

TABLE 5: List of influence on index systems and weight of medium and large marathon events.

\begin{tabular}{|c|c|c|c|c|c|}
\hline $\begin{array}{l}\text { Primary } \\
\text { indicators }\end{array}$ & Weight & Secondary indicators & Weight & Tertiary indicators & Weight \\
\hline \multirow{4}{*}{ Polity A1 } & \multirow{4}{*}{0.179} & \multirow{3}{*}{ Policy implementation B1 } & \multirow{3}{*}{0.094} & Implementation degree of national fitness $\mathrm{C} 1$ & 0.028 \\
\hline & & & & The practice degree of the new pattern of the competition C2 & 0.031 \\
\hline & & & & $\begin{array}{l}\text { Construction intensity of building a strong city with sports } \\
\text { talents C4 }\end{array}$ & 0.029 \\
\hline & & Regional exchanges B2 & 0.085 & Regional event exchange C7 & 0.027 \\
\hline \multirow{4}{*}{ Economy A2 } & \multirow{4}{*}{0.176} & & & Regional per capita sports GDP C9 & 0.030 \\
\hline & & Positive economic impact B3 & 0.089 & Local event organizers C10 & 0.028 \\
\hline & & & & Economic benefits of regional characteristic brand $\mathrm{C} 11$ & 0.026 \\
\hline & & $\begin{array}{l}\text { Negative economic impact } \\
\text { B4 }\end{array}$ & 0.087 & Positive impact on tourism $\mathrm{C} 13$ & 0.027 \\
\hline \multirow{8}{*}{ Culture A3 } & \multirow{8}{*}{0.185} & \multirow{8}{*}{$\begin{array}{l}\text { Carry forward the } \\
\text { characteristics B6 }\end{array}$} & \multirow{3}{*}{0.096} & Carry forward the Chinese spirit of sports C21 & 0.028 \\
\hline & & & & The development of sports spirit and cultureC24 & 0.032 \\
\hline & & & & Event-related cultural events C25 & 0.030 \\
\hline & & & \multirow{5}{*}{0.089} & Publicity of the featured events C26 & 0.032 \\
\hline & & & & Design of characteristic medals C27 & 0.027 \\
\hline & & & & Design of characteristic competition route C28 & 0.026 \\
\hline & & & & Setting of characteristic events C29 & 0.028 \\
\hline & & & & Display of regional culture C30 & 0.026 \\
\hline \multirow{14}{*}{ Society A4 } & \multirow{14}{*}{0.373} & \multirow{4}{*}{ Regional prestige $\mathrm{B} 7$} & \multirow{4}{*}{0.090} & Event level C31 & 0.032 \\
\hline & & & & Media publicity C32 & 0.030 \\
\hline & & & & Athletes' satisfaction C33 & 0.028 \\
\hline & & & & Sponsor satisfaction C34 & 0.024 \\
\hline & & \multirow{4}{*}{ Event visibility B8 } & \multirow{4}{*}{0.090} & Number of contestants level C35 & 0.023 \\
\hline & & & & Level of the contestant C36 & 0.032 \\
\hline & & & & Media quantity and level C37 & 0.029 \\
\hline & & & & Regional marathon participation C38 & 0.032 \\
\hline & & \multirow{4}{*}{$\begin{array}{l}\text { Regional sports } \\
\text { popularization B9 }\end{array}$} & \multirow{4}{*}{0.099} & Regional sports event participation C39 & 0.031 \\
\hline & & & & Regional citizens pay attention to the ways $\mathrm{C} 40$ & 0.028 \\
\hline & & & & Regional citizens' sports literacy training C41 & 0.033 \\
\hline & & & & Level of urban sports public service C42 & 0.031 \\
\hline & & \multirow{2}{*}{$\begin{array}{l}\text { Educational diffusion effect } \\
\text { B11 }\end{array}$} & \multirow{2}{*}{0.094} & Research on university marathon topics C47 & 0.032 \\
\hline & & & & Number of heavy horse research topics C48 & 0.027 \\
\hline
\end{tabular}


TABle 5: Continued.

\begin{tabular}{|c|c|c|c|c|c|}
\hline $\begin{array}{l}\text { Primary } \\
\text { indicators }\end{array}$ & Weight & Secondary indicators & Weight & Tertiary indicators & Weight \\
\hline \multirow{7}{*}{ Environment A5 } & \multirow{7}{*}{0.087} & \multirow{4}{*}{$\begin{array}{l}\text { Positive environmental } \\
\text { impact B12 }\end{array}$} & \multirow{4}{*}{0.071} & $\begin{array}{l}\text { Implementation of precompetition environmental publicity } \\
\qquad 50\end{array}$ & 0.164 \\
\hline & & & & Environmental remediation caused by the event C51 & 0.170 \\
\hline & & & & $\begin{array}{l}\text { The implementation of the event in environmental } \\
\text { demonstration C52 }\end{array}$ & 0.164 \\
\hline & & & & $\begin{array}{c}\text { Promotion of the event to local residents' awareness of } \\
\text { environmental protection C53 }\end{array}$ & 0.164 \\
\hline & & \multirow{3}{*}{$\begin{array}{l}\text { Negative environmental } \\
\text { impact B13 }\end{array}$} & \multirow{3}{*}{0.016} & Environmental protection facilities C54 & 0.152 \\
\hline & & & & $\begin{array}{l}\text { Degree of damage of the surrounding living environment } \\
\text { C56 }\end{array}$ & 0.096 \\
\hline & & & & Waste cleaning efforts C57 & 0.091 \\
\hline
\end{tabular}

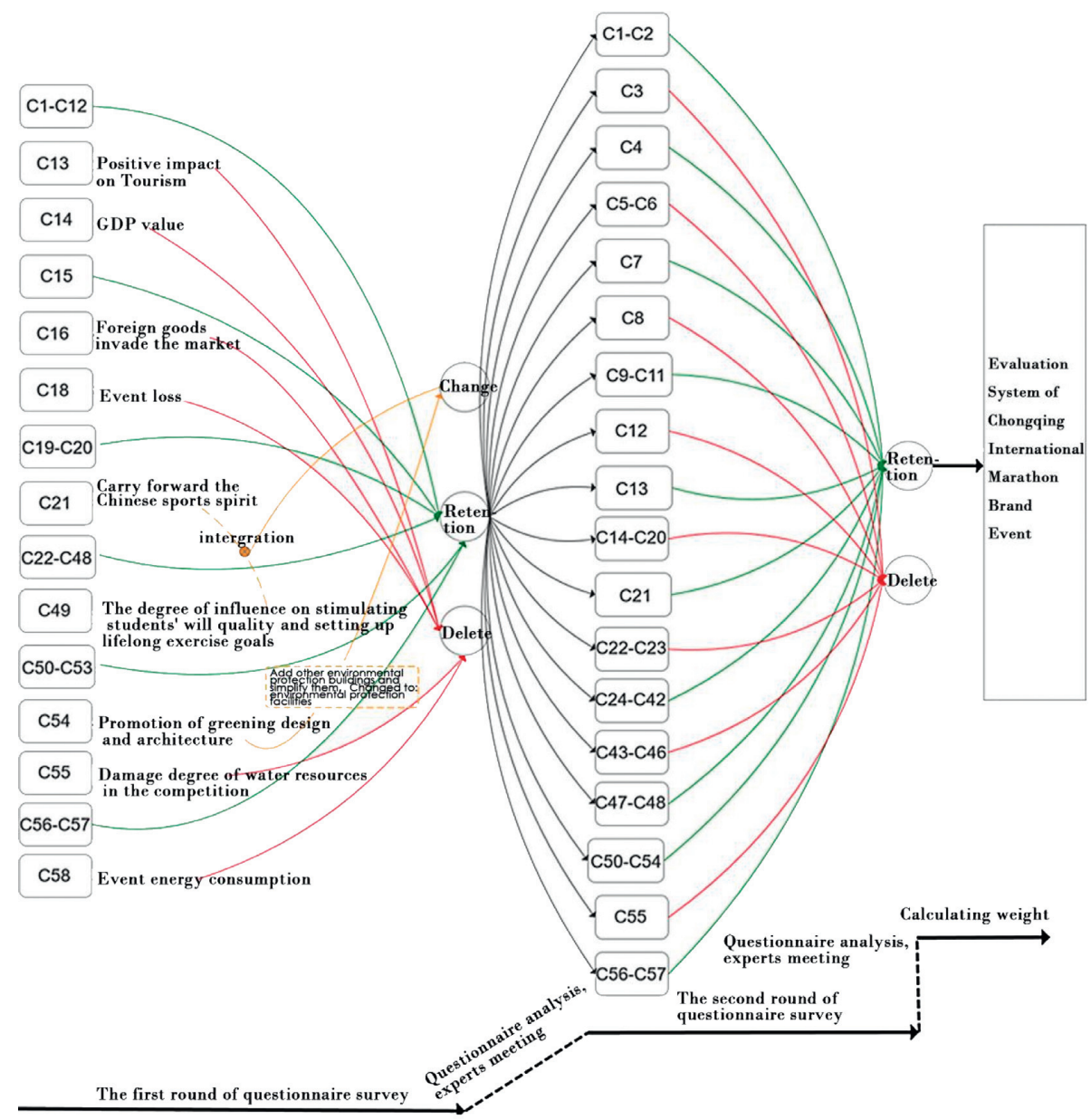

FIgURE 2: Index deletion and establishment of the evaluation system. 


$$
X=\int x f(x) \mathrm{d} x
$$

among them,

$$
f(x)= \begin{cases}\frac{1}{b-a}, & 0<x<a, \\ 0, & \text { others }\end{cases}
$$$$
b=5, a=1 .
$$

The calculation method of the weight coefficient of tertiary indicators is as follows: (1) calculating the sum of expert scoring weights of all tertiary indicators $\sum_{m=1}^{35} X_{m}$ and (2) weighting coefficient of the $m$-th tertiary indicators $W_{m}=\left(X_{m} / \sum_{m=1}^{35} X_{m}\right)$.

The calculation method of the weight coefficient of secondary indicators is as follows: (1) calculating the expert scoring weight $X_{i}$ of each secondary indicators, respectively, (2) calculating the sum of all expert weights of all secondary indicators $\sum_{i=1}^{11} X_{i}$, and (3) weighting coefficient of the $i$-th secondary indicators $W_{i}=\left(X_{i} / \sum_{i=1}^{11} X_{i}\right)$.

The calculation method of the weight coefficient of primary indicators is as follows: (1) calculating the expert scoring weight $X_{j}$ of each primary indicator, respectively, (2) calculating the sum of expert weights of all primary indicators $\sum_{j=1}^{5} X_{j}$, and (3) weighting coefficient of the $j$-th primary indicators $W_{j}=\left(X_{j} / \sum_{j=1}^{5} X_{j}\right)$.

\section{Conclusions and Outlooks}

5.1. Conclusions. As one of the top marathon brand events in Southwest China and even at home and abroad, the Chongqing International Marathon itself has its unique research value. Therefore, the construction of a complete set of logical, highly scientific, and rigorous index evaluation system for the Chongqing International Marathon brand events is conducive to the comprehensive impact of the event itself on the host region and provides important theoretical support for the development of the Chongqing International Marathon brand events and the practical work of relevant sports departments in the future. It also has more practical significance.

The core of the Chongqing International Marathon is a sports competition, and the event itself is located in Chongqing. Since its establishment, it has always been a characteristic event of Chongqing mountain city and river water. Therefore, building such a marathon brand event in line with Chongqing will have a positive impact on the regional politics, economy, culture, society, and environment and will also become a landmark social activity in Chongqing.

In this study, the evaluation index system of the Chongqing International Marathon brand competition established by using the methods of literature, Delphi, and analytic hierarchy process includes 5 primary indicators, 13 secondary indicators, and 58 tertiary indicators, including politics, economy, culture, society, and environment. Through the evaluation index system of the Chongqing
International Marathon brand event, it can be seen that society (0.523) is the main impact of the event on the host region, indicating that the event itself has a great impact on all levels of society in the host region; then, the event also has a great impact on the regional culture (0.519), indicating that the event plays a positive role in promoting and carrying forward culture; second, the impact of the event on the politics (0.504) and economy (0.496) of the host region is roughly the same; finally, the relatively small impact of the event on the local environment (0.487) shows that with the event held for many years, people have formed a good awareness of environmental protection.

The advantage of the research method adopted in this study is that it combines both subjective research methods (Delphi method) and objective research methods (AHP), because it avoids greater subjectivity compared to only using the Delphi method for research. Compared with the method that only uses survey data, it avoids overanalysis of data.

5.2. Outlook. The brand event evaluation index system constructed in this study takes the Chongqing International Marathon as the research object. The index is relatively detailed and contains strong integrity. However, in the process of consultation and interview, due to limited conditions, only 15 experts and scholars' opinions were collected, and only 15 experts were consulted in the overall study. The number of experts is relatively small. Due to the large scope of the research object, the selection of experts is still not comprehensive. In addition, there is little research on the construction of the marathon brand event evaluation system in China, which has a certain impact on the construction of the Chongqing International Marathon event evaluation system. However, the author of this study will continue this kind of research and will further improve and improve in many deficiencies.

\section{Data Availability}

No data were used to support this study.

\section{Conflicts of Interest}

The authors declare no conflicts of interest.

\section{Acknowledgments}

The authors would like to thank Mr. Li and Mr. Zhang of the Organizing Committee of Chongqing International Marathon for their support for our research work. Their data and help for our research enabled the authors to have first-hand accurate data in the writing of the paper and ensure the authenticity of the paper. At the same time, the authors would like to thank the experts and scholars interviewed in the paper, who put forward their valuable opinions and comments on the construction of the evaluation system in their busy schedule, so that they can improve the construction of the evaluation system step by step and make the framework of the whole paper more rigorous. Finally, the authors would like to thank Mr. Jin, Mr. Teng, Mr. Huang, 
and Mr. Li of the research group for their full participation in the Chongqing International Marathon. Without the joint efforts of the research group, there would be no delivery of today's paper. Here, the authors would like to express their heartfelt thanks to the teachers who have guided and helped them! At the same time, the authors would also like to thank the monographs of scholars cited in this paper. Without the inspiration and help of the research results of these scholars, the authors would not be able to complete the final writing of this paper. The authors also want to thank their friends and classmates. They gave them a lot of useful materials in the process of writing this paper and also provided warm help in the process of typesetting and writing this paper! There are spots even in the sun. Due to their limited academic level, this thesis will inevitably have shortcomings. The authors urge all teachers and students to criticize and correct. This work was supported by the Deanship of Scientific Research at the King Saud University for funding this work through research group no. RG-1438-027; general project of Chongqing Sports Bureau in 2020: research on the construction of evaluation system of Chongqing International Marathon brand competition (project no.: d202017); and social science fund project of the Chongqing University of Posts and Telecommunications (project no.: k2020-84).

\section{References}

[1] X. Wang, "Research on media image communication of sports organizations-taking 2019 Chongqing International Marathon as an example," Global Premiere, vol. 4, no. 4, Article ID $115,2019$.

[2] D. Li, "The impact of Chongqing International Marathon on Chongqing's urban development," China Sports Science Society, vol. 2, 2017.

[3] Li Guoqiang, R. Wang, H. Xu, and Y. Wen, "Construction and empirical research on mechanism model of marathon development in China," Journal of Shenyang Institute of Physical Education, vol. 40, no. 2, pp. 67-76, 2021.

[4] T. Tao, "Construction of the evaluation index system of government-supported mass sports events," Construction of the evaluation index system of government-supported mass sports events, vol. 11, p. 2, 2019.

[5] Z. Li, "Research on the construction of evaluation index system of mountain outdoor sports events," Master's degree thesis, China University of Geosciences, Beijing, China, 2019.

[6] A. Xu, "Application of Delphi method and its difficulties," China Statistics, vol. 1, no. 9, 2006.

[7] J. Guo, Z. Zhang, and Q. Sun, "Research and application of analytic hierarchy process," China Safety Science Journal, vol. 18, no. 5, pp. 148-153, 2008.

[8] J. Zheng and J. Huang, "The Construction of evaluation index system of basic public sports service," Journal of Shanghai Physical Education Institute, vol. 37, no. 1, pp. 9-13, 2013.

[9] M. Bao, Promoting the High-Quality Development of Sports in the 14th Five Year Plan under the Guidance of Building a Sports Power in an All-Round Way, China Sports Daily, Beijing, China, 2020.

[10] Y. Liang, "Advantage perspective and system theory: two perspectives of social work," Sea of Learning, vol. 4, pp. 70-78, 2013.

[11] Y. Chen, “On the difference between von bertalanffy' general system theory and santa Fe institute's complex adaptive systems theory," Journal of Shandong University of Science and Technology, vol. 2, pp. 5-8, 2007.

[12] C. Luo, "Research on the evaluation system of taiyuan international marathon," Master's degree thesis, University Of Shanxi, Taiyuan, China, 2012.

[13] W. Du, "Connotation and index construction of a sports power," M.S. thesis, Master's degree, Guangzhou Institute of Physical Education, 2020.

[14] L. Qi, "Statistical analysis and fuzzy comprehensive evaluation of the Richter scale," Shandong Science, vol. 2, pp. 18-23+28, 2006.

[15] Hodley, L. Liu, Y. Yuan, X. Chen, and B. Zhao, "Study on the weight of Beijing Winter Olympics based on entropy method," Journal of Shenyang Institute of Physical Education, vol. 38, no. 5, pp. 47-55, 2019. 